\title{
Research Paper \\ Efficacy of Mindfulness-Based Cognitive Therapy on Headache Considering the Moderating Role of Alexithymia: A Randomized Controlled Trial
}

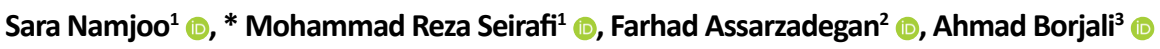

1. Department of Health Psychology, Faculty of Psychology, Karaj Branch, Islamic Azad University, Karaj, Iran.

2. Department of Neurology, School of Medicine, Shahid Beheshti University of Medical Sciences and Health Services, Tehran, Iran.

3. Department of Clinical Psychology, Faculty of Psychology \& Educational Sciences, Allameh Tabataba'i University, Tehran, Iran.

\begin{tabular}{|c|c|}
\hline $\begin{array}{l}\text { Use your device to scan } \\
\text { and read the article online }\end{array}$ & dtration Namjoo S, Seirafi MR, Assarzadegan F, Borjali A. Efficacy of Mindfulness-Based Cognitive Therapy on Headache \\
\hline$\square+\square$ & Considering the Moderating Role of Alexithymia: A Randomized Controlled Trial. The Journal of Qazvin University of Medical \\
\hline 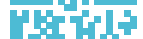 & Sciences. 2019; 22(6):150-163. https://doi.org/10.32598/JQUMS.22.6.150 \\
\hline a & dol'https://doi.org/10.32598/JQUMS.22.6.150 \\
\hline
\end{tabular}

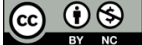

Received: 11 Aug 2018 Accepted: 03 Oct 2018 Available Online: 01 Feb 2019

Keywords: Mindfulness-Based Cognitive Therapy, Primary headache, Alexithymia

\begin{abstract}
A B STRACT
Background Mindfulness-based intervention has been found efficacious in reducing primary headaches and negative cognitive-related pain. However, little is known about the potential moderators in this regard. One of the most important moderators affecting the headaches is Alexithymia.

Objective The present study investigated the moderating role of Alexithymia on the effect of Mindfulness-Based Cognitive Therapy (MBCT) on pain intensity in patients with primary headache.

Methods A clinical trial was conducted in 2017 and 2018 at Imam Hossein Hospital of Tehran City, Iran. Using judgment sampling method, of 94 inpatients with chronic headache, 85 were selected and randomly assigned into the two groups of MBCT (43 patients) and control (42 patients). The Numerical Rating Scale (NRS) of Pain Self-Efficacy Questionnaire (PSEQ) and Pain Catastrophizing Scale (PCS) were applied to collect data. The obtained data were analyzed by Independent Samples t-test and Chi-squared test; longitudinal data were analyzed using linear mixed model analysis.

Findings Statistically significant timexgroup interactions were found for pain intensity $(P<0.001)$, selfefficacy $(P<0.001)$, and catastrophizing $(P<0.001)$. The obtained results indicated a significant reduction in pain, pain catastrophizing and increased self-efficacy.

Conclusion MBCT is a potentially efficacious approach for individuals with headache. Alexithymia may have clinically relevant factors for identifying the patients who may benefit most from MBCT as a pain intervention.
\end{abstract}

\section{Extended Abstract}

\section{Introduction}

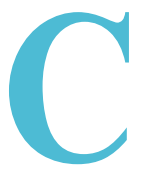

hronic pain is a prevalent annoying condition in humans. Primary headaches are among the most commonly reported complaints in neurological clinics leading to high annual healthcare costs [1]. Studies using applied technologies such as Magnetic Resonance Imaging (MRI) have reported that in the brain, the sensitive and important paths related to pain sensation pass along the cognitive and emotional paths. Thus, such neuromatrix have the capacity to increase or decrease the sensory flow of painful stimuli through cognitive activities [2].

Such studies have suggested that psychological processes can actually develop painful stimulus pathways in the brain;

\section{* Corresponding Author:}

Mohammad Reza Seirafi, PhD.

Address: Department of Psychology, Faculty of Psychology, Karaj Branch, Islamic Azad University, Karaj, Iran.

Tel: +98 (26) 34182657

E-Mail: seirafihealth@gmail.com 
thus, provide evidence that psychological interventions have a strong potential for chronic pain management. Numerous studies have revealed that ineffective cognitive skills play a key role in the persistence of negative emotions and initiate maladaptive behavioral responses, resulting in continued pain-related outcomes [3]

One of the treatments that target negative cognition is Mindfulness-Based Cognitive Therapy (MBCT), recognized in several studies conducted over the past 15 years of effective pain relief [4-8]. A modern psychological approach (MBCT) has recently devised a comprehensive model to explore the impact of cognitive process on perception and pain management, specifically for chronic pain [9]. Randomized clinical trials indicated the effectiveness of MBCT on chronic pain $[10,11]$.

Individual differences in the clinical profiles of psychological traits in patients with chronic pain have been proposed as one of the potential sources of various responds to treatment $[12,13]$. However, little attention has been paid to the association between individual characteristics and MBCT, as well as specific moderators that maximize treatment outcomes. Alexithymia is identified as a key factor in chronic headache. Alexithymia is a persistent personality trait, defecting the cognitive processing of emotions. Additionally, high levels of Alexithymia may predict headache severity [14].

This clinical feature is associated with the inability to identify and name the emotions, as well as pain severity in patients [15]. Its high levels are considered as one of the headache severity predictors [16]. It is assumed during mindfulness practices (due to interaction with body sensations and affective distress) emotions are cognitively processed [17]. Alexithymia can be considered as one of the predictors of mindfulness-based interventions effects on headache. Some clinical trials have indicated the moderating role of Alexithymia on therapeutic outcomes [18]. However, little is known about the moderating role of Alexithymia in the
MBCT effects [19]. Therefore, based on the aforementioned contents, the present study investigated the effect of MBCT on pain intensity and the moderating role of Alexithymia on the same effect in patients with primary headache.

\section{Methods and Materials}

Data from the current experimental study is extracted from a single blind Randomized Controlled Trial (RCT) that was performed to compare the effect of MBCT with Attention Placebo Control (APC) group. The sample population consisted of 85 patients with a primary headache referring to the Neurology Department of Imam Hossein Hospital. Of 94 eligible participants, 4 have discontinued the research, 5 people did not attend the baseline assessments and the remaining 85 participants (after completing the baseline questionnaire using the random sequencing website $\left.{ }^{1}\right)$ were allocated to treatment (MBCT, n=43) and control (APC, n=42) groups.

In the experimental group, 6 participants discontinued the study before the intervention and 9 participants discontinued it during the intervention. Thus, a total of 28 people completed the questionnaire by the end of the eighth week of intervention. In the control group, 8 cases were missed and by the end of the eight weeks, 34 patients remained in this group. To measure changes related to the duration of treatment, the study participants were evaluated at 4 stages from the onset of treatment to the end of the eight weeks. SPSS was used for data analysis.

To evaluate the efficacy of pain intensity, self-efficacy and pain catastrophizing and its comparison with the APC group, Linear Mixed Models were used. At first, the interactive effect of time (4 steps) $\times$ group (MBCT and APC groups) was obtained to evaluate the effectiveness of MBCT. Then, the interactive effect of the moderator variable (Alexithymia) $\times$ time (4 steps) $\times$ group (MBCT and APC groups) was investigated

1. http://www.randomizer.org

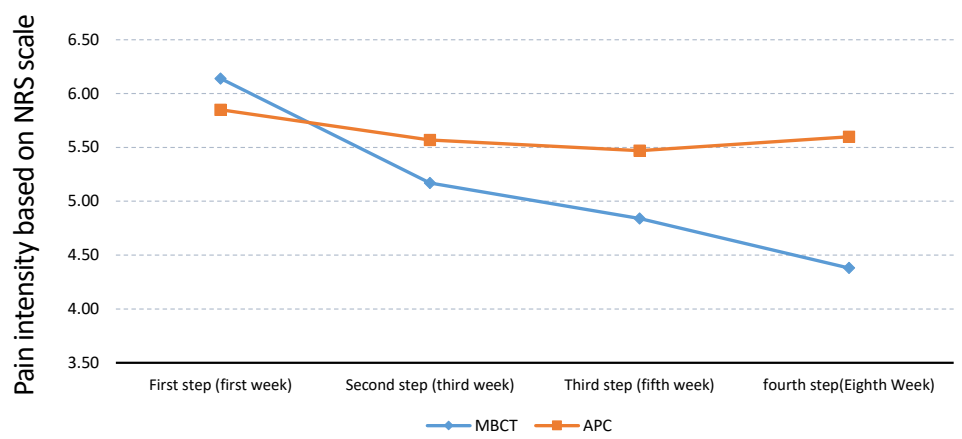

Qazvin University of Medical Sciences (JOUMS)

Figure 1. Raw score's changes in pain intensity from baseline to the end of the eighth week (measured in 4 sessions) 


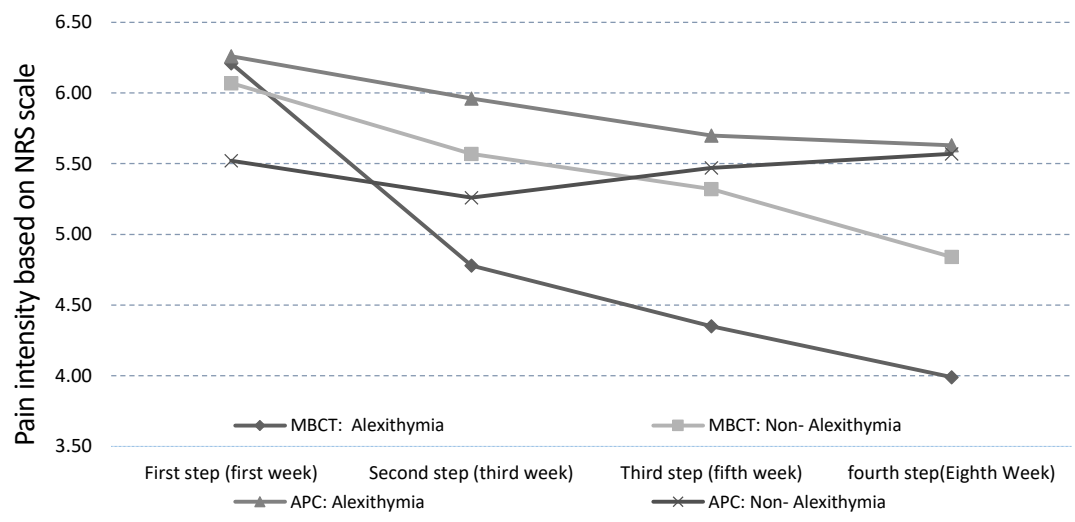

Figure 2. Inter-group results of interactive effects of Alexithymia on pain (Time $\times$ Group $\times$ Moderator) $\begin{gathered}\text { The Jurnal of } \\ \text { Oazvin University of Medical Sciences (Joums) }\end{gathered}$

\section{Results}

The interactive effect of treatment group $\times$ time in all variables was only significant in the MBCT group $(\mathrm{P}<0.001)$. The pain intensity changes are shown in Figure 1. According to Figure 2, this significant difference is explained by the trend of pain reduction from the second assessment step (the third week of intervention) with a steep slope to the cut-off point of the middle pain (numerical value: 5 ); then has been reduced until the last assessment step in the alexithymic participants of MBCT group.

The non-alexithymic participants of MBCT group have reached moderate pain level in the fourth step with a mild slope. It indicates that MBCT gradually improves the condition of healthy and non-alexithymic participants. Moreover, these groups have obtained statistically significant results compared to the control group; however, after receiving MBCT sessions, they demonstrated less therapeutic benefits than those with Alexithymia.

\section{Conclusion}

It is important to distinguish between the individuals with and without Alexithymia. Such distinction must be considered in the treatment trend of chronic pain management. In addition, the obtained results can explain the effect of MBCT on adjustment with primary headache.

\section{Ethical Considerations}

\section{Compliance with ethical guidelines}

This study was approved by the Ethics Committee of Islamic Azad University, Karaj Branch (Ethical code: IR.IAU.K.REC.1397). The Iranian Center for Clinical Trials has registered this study with the code IRCT20141012019511N4.
Funding

The financial support was provided by Islamic Azad University, Karaj Branch.

\section{Authors' contributions}

Study concept and design: Sara Namjoo, Mohammad Reza Seirafi; Original Draft, Analysis and interpretation of data: Sara Namjoo; Manuscript preparation: Sara Namjoo, Mohammad Reza Seirfi, Ahmad Borjali, Farhad Assarzadegan; Data collection: Mohammad Reza Seirafi, Ahmad Borjali, Farhad Assarzadegan; Critical revision: Ahmad Borjali, Mohammad Reza Seirafi.

\section{Conflicts of interest}

The authors declared no conflict of interest.

\section{Acknowledgments}

We are grateful for the cooperation and assistance of the Research Deputy, the officials and members of the Imam Hossein Hospital and the executive staff. 


\title{
اثر شناختدرمانى مبتنى بر ذهن آتاهى بر سردرد اوليه: يك كارآزمايى بالينى تصادفى با بررسى تعديل ترَى الكسيتايميا
}

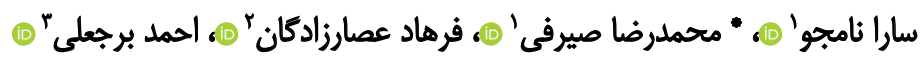 \\ 1- كروه روانشناسى سلامت، دانشكده روانشناسى، واحد كرج، دانشكاه آزاد اسلامى، كرجه ايران.

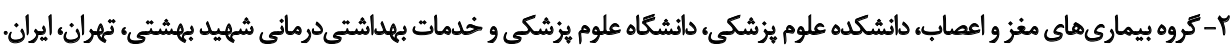

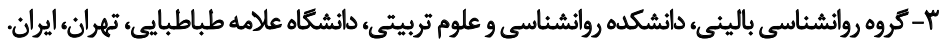

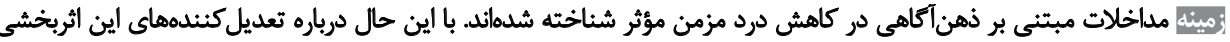

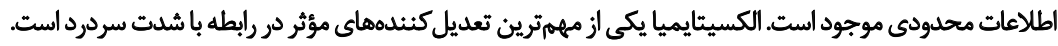

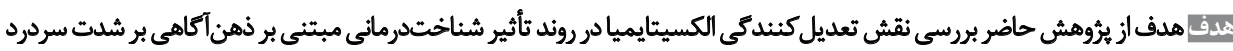
اوليه بود.

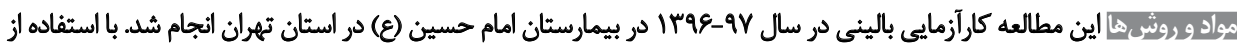

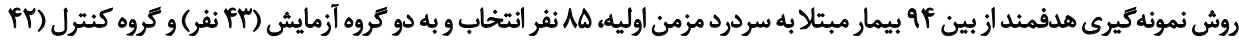

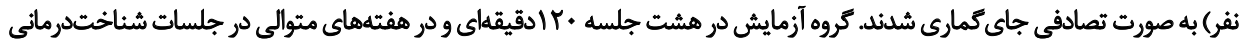

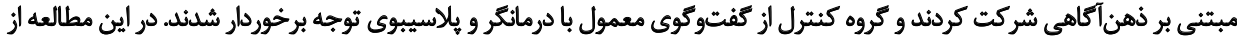

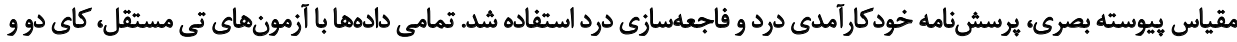

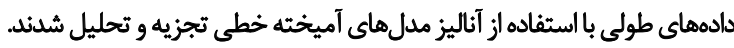

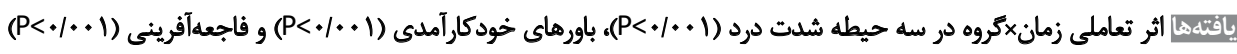

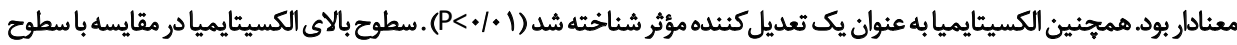

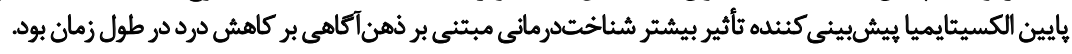

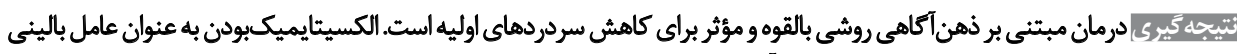

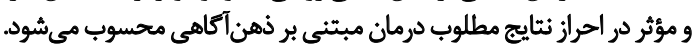

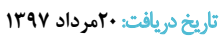
تاريخ هذيرش: الريخ

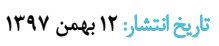

كليدواروهائ

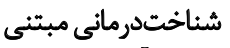

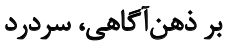

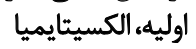

با استفاده از تكنولوريىهاى كاربردى مانند امآرآى مشاهده شده

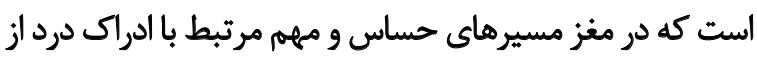

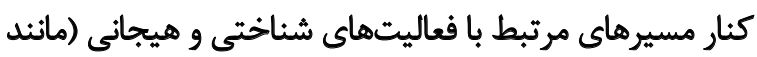

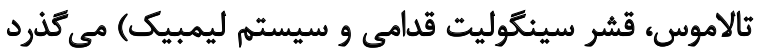

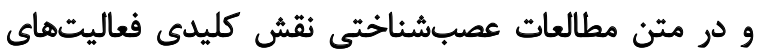

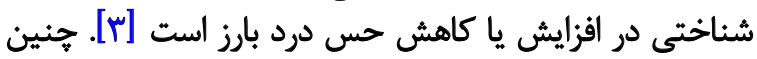

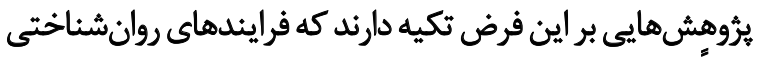

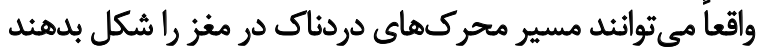

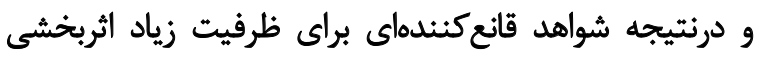

رردرد يكى از رايجترين شكايتهاى بروسى اسشده در

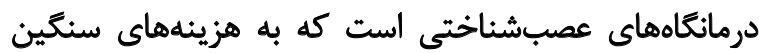

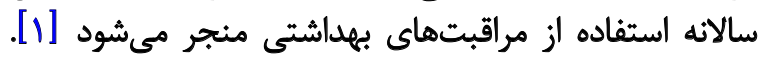

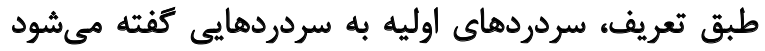

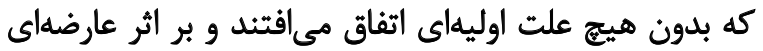

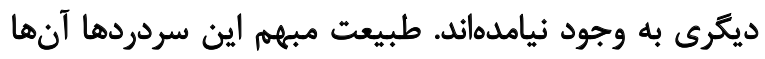

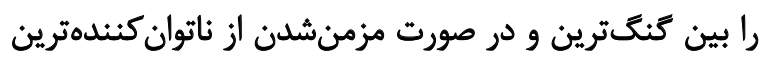

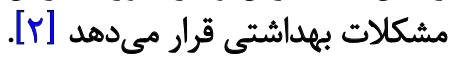


با اين فرض كه در ذهن آكاهى (به واسطه ارتباط برقراركردن

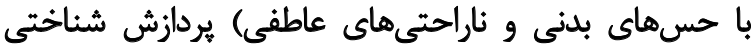

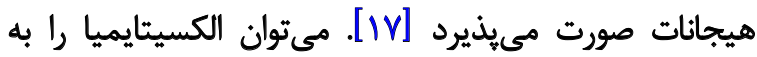

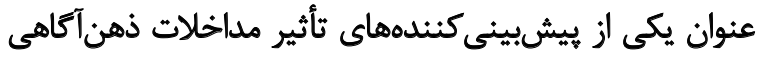

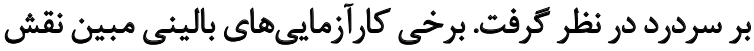

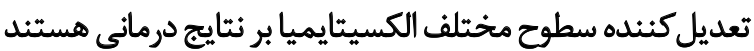

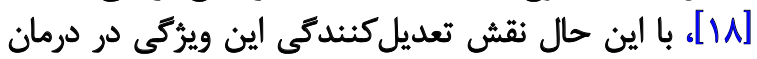

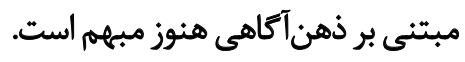

در آخر، باوجود يثوهشى هاى مقدماتى حاكى از اثربخشبوني

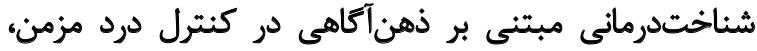

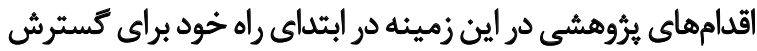

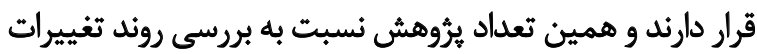

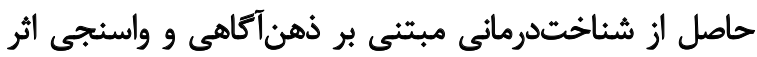

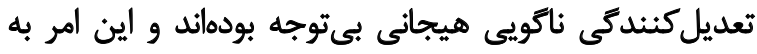

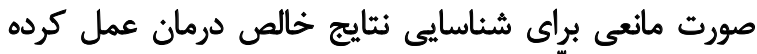

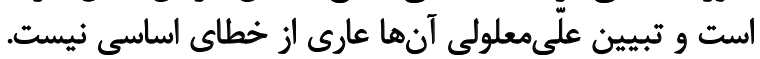

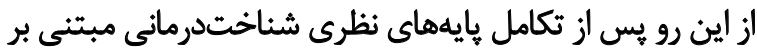

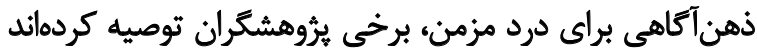

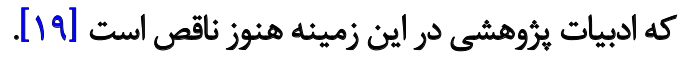

همجنين به نظر ميرسد بازتابدادن نتايج خالص اثربخشى بردي

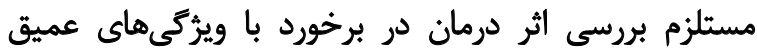

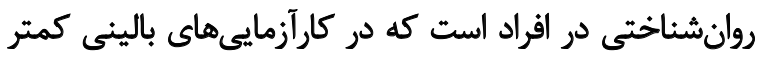

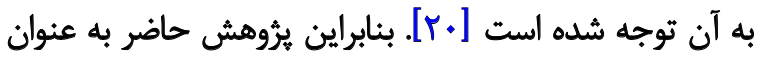

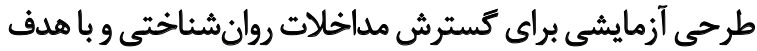

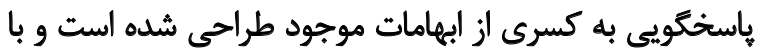

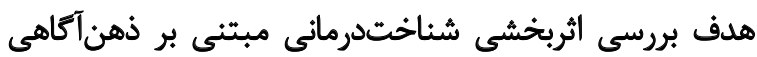

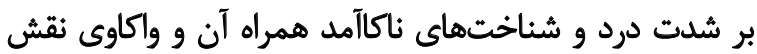

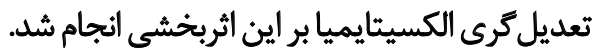

\section{مواد و روش مها}

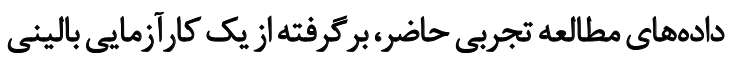

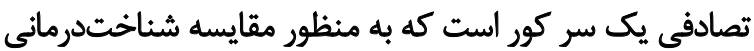

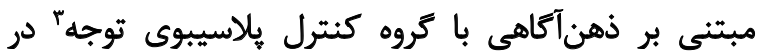

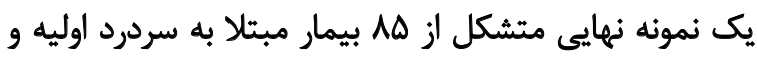

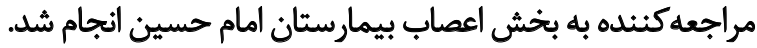

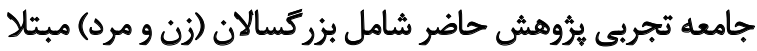

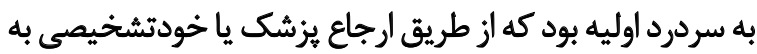

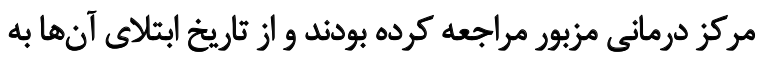

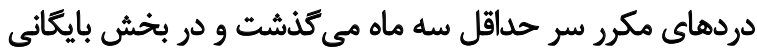

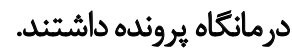

معيارهاى ورود به مطالعه عبارت بودند از: سن بيشتر از 19

3. Attention Placebo Control group (APC)
مداخلات روانشناختى در درمان دردهاى مزمن ارائه كرداند. از

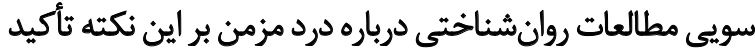

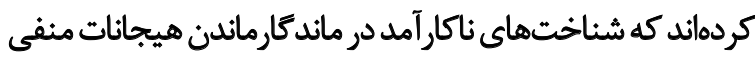

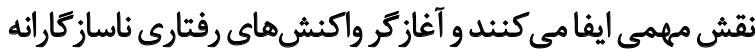

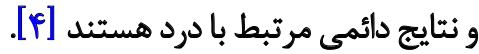
در بررسى متآناليز مطالعات انجامشده در هان سال كذشته

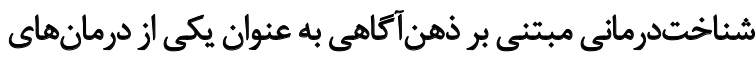

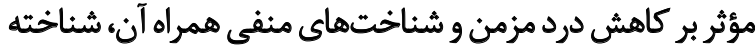

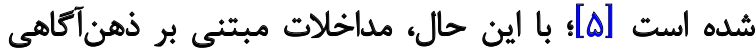

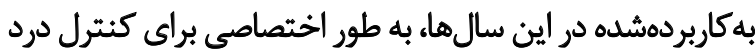

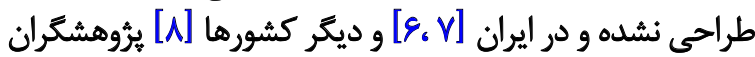

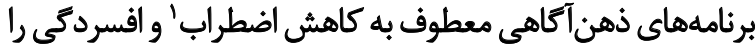

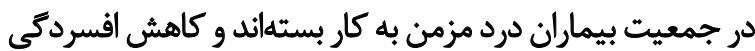

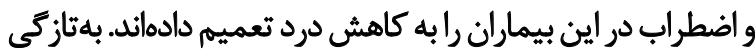

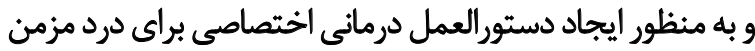

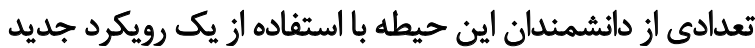

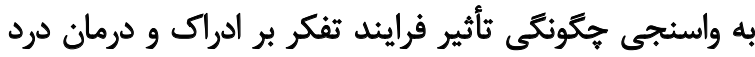

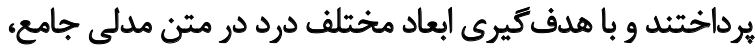

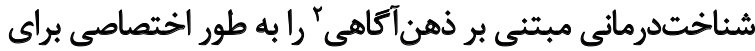

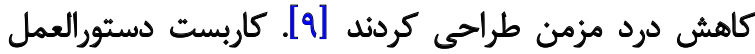

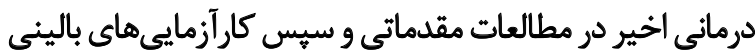

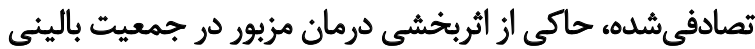

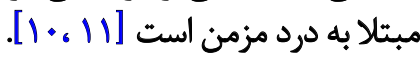

در اين مسير، ابهام كشايى از ميزان واقعى اثربخشى مداخلات

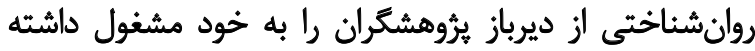

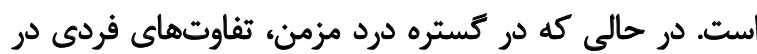

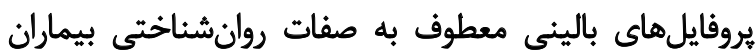

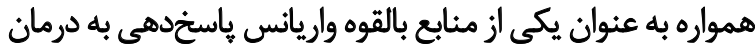

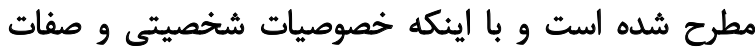

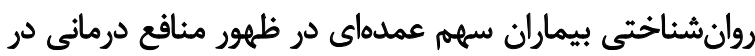

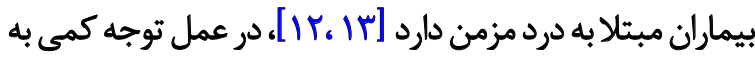

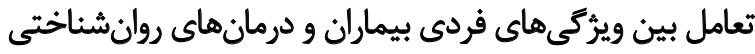

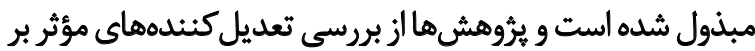

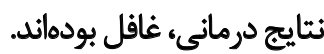

در اين بين يكى از صفات روانشناختى مؤثر بر درد مزمن،

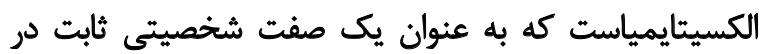

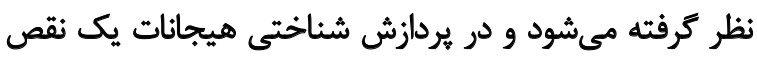

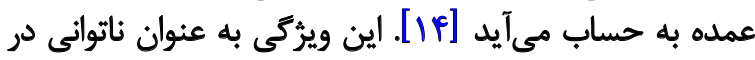

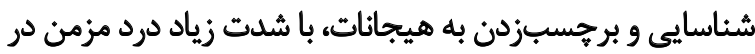

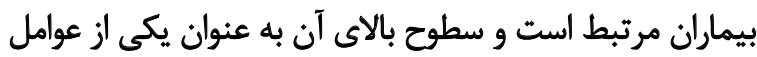

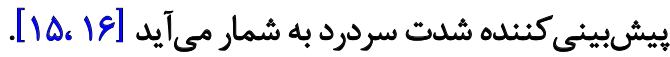


درباره اطلاعات بالينى مربوط به سردرد با استفاده از روزنتار

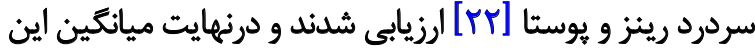

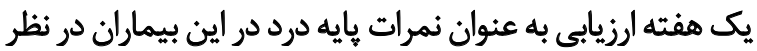
كرفته شد.

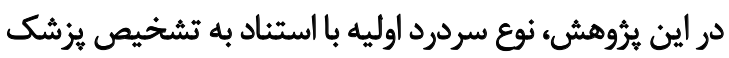

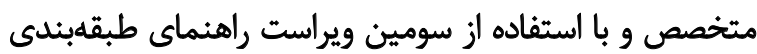

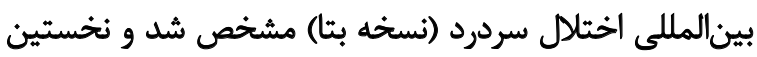

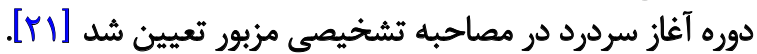

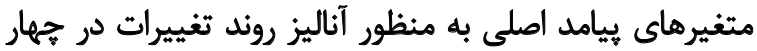

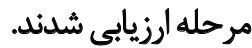

متغير شدت درد با استفاده از مقياس الادرجهاى دردي"

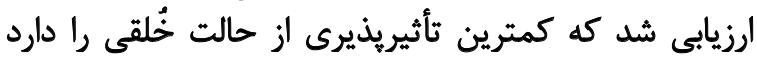

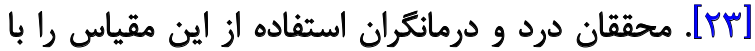

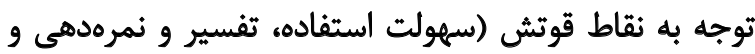

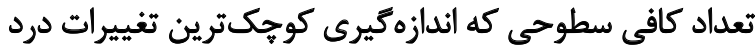

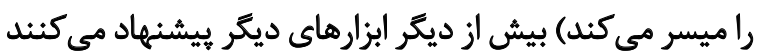

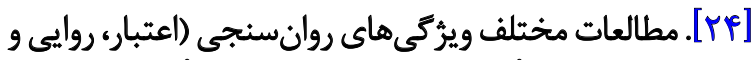

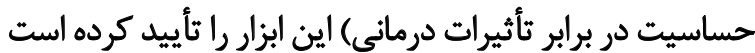

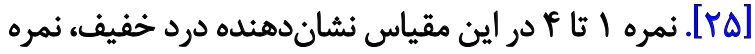

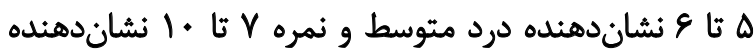

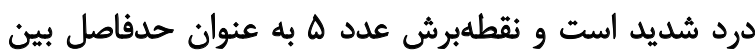

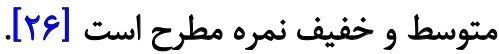

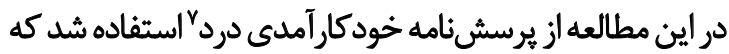

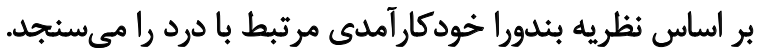

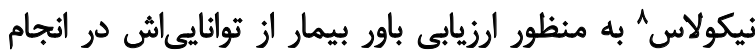

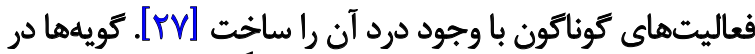

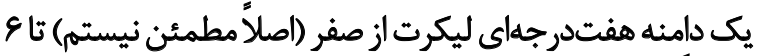

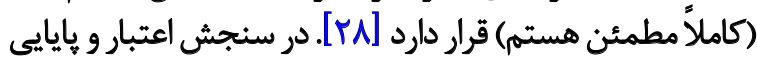

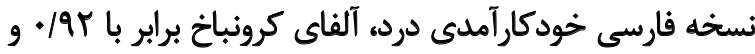

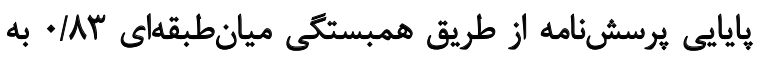

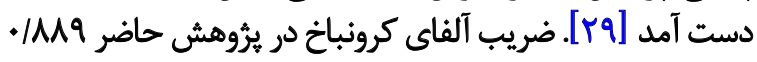
به دست آمده است.

از مقياس فاجعلسازى درد" براى شناختهاى فاجعآهآميز

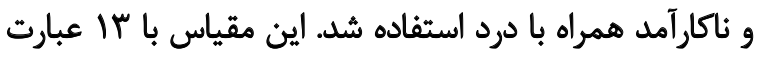

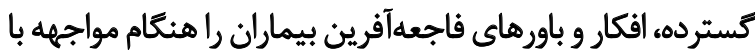

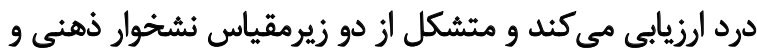

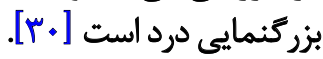

هريك از عبارات اين يرسشنامه بر اساس يك مقياس

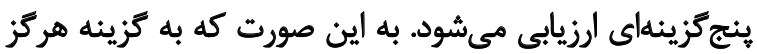

6. Numeric Rating Scale (NRS)

7. Pain Self Efficacy Questionnaire (PSEQ)

8. Nicholas

9. Pain Catastrophizing Scale (PCS)
سال؛ داشتن تجربه سردرد بيش از ب روز در مر ماه (به مدائ

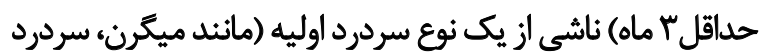

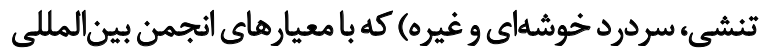

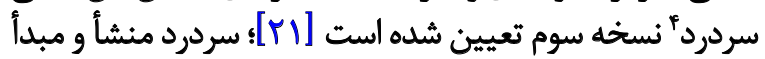

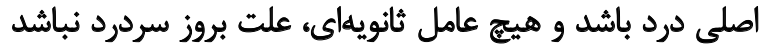

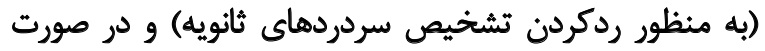

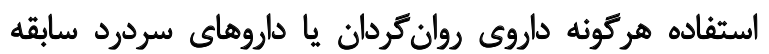

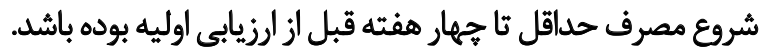

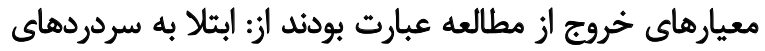

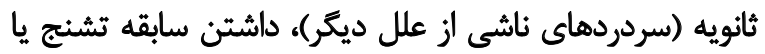

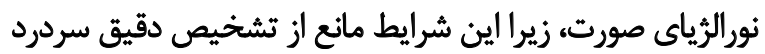

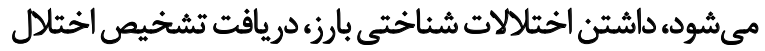

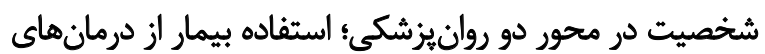

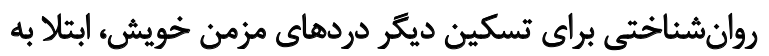

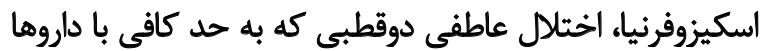
كنترل نشده است يا مصرف مواد مخدر.

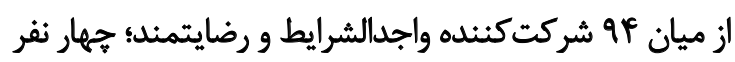

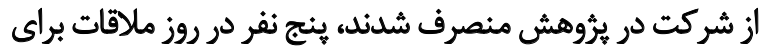

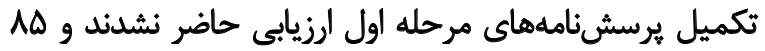

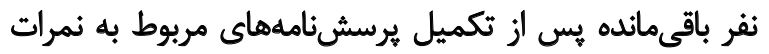

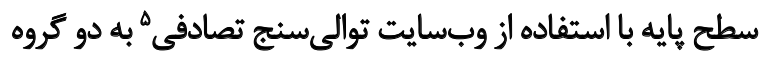

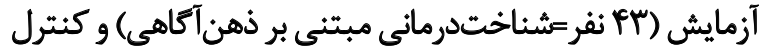

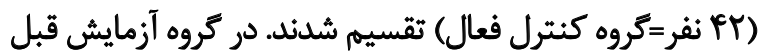

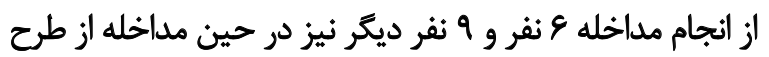

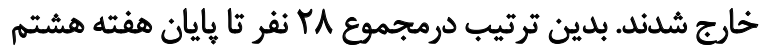

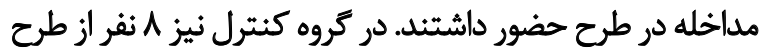

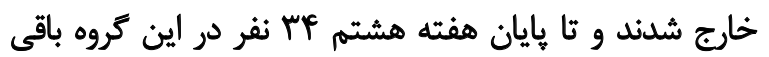

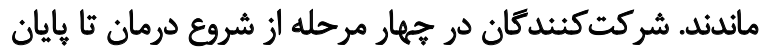

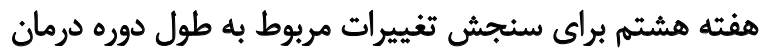

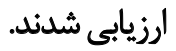

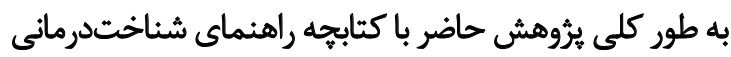

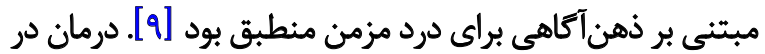

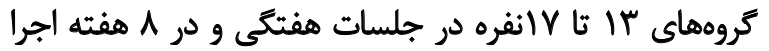

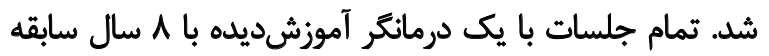

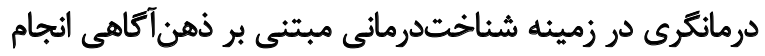

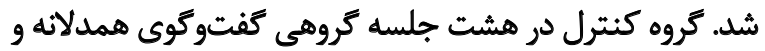

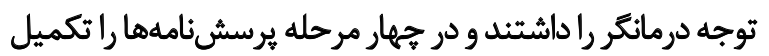

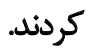

اطلاعات جامعهشناختى همه بيماران جمعآورى شد و از آنجا

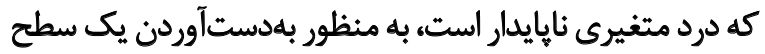

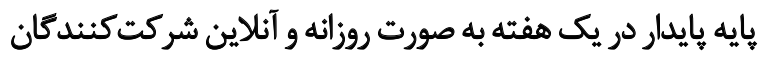

4. International Headache Society (IHS)

5. http://www.randomizer.org 
تعاملى متغير تعديلكر (الكسيتايميا)جزمان (جهار مرحله) ×كروه

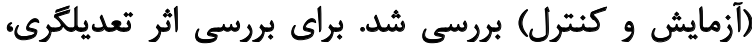

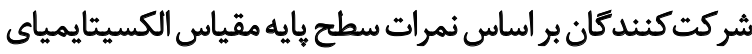

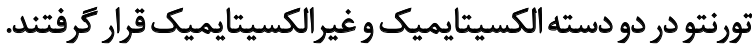

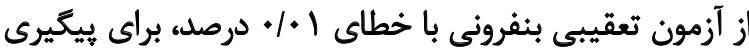
اختلاف بين كروهها استفاده شد.

در اين ثروهش براى برخورد با تمشدگى دادهها در سطح

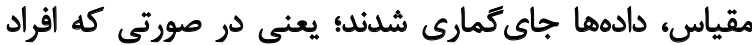

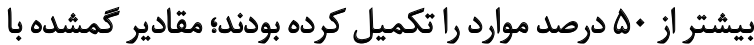

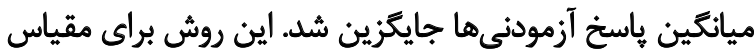

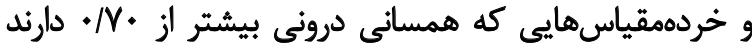

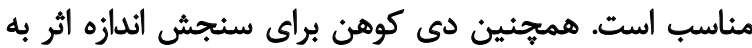

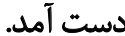

Lation

از نظر آمارى در هيجيك از متغيرهاى جمعيتشناختي

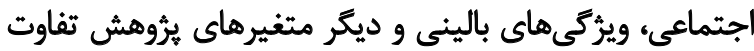

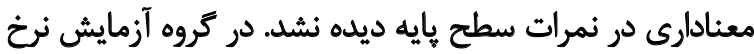

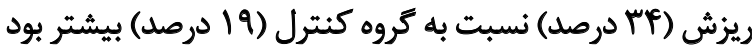

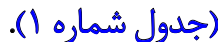

درباره متغير شدت درده اثر زمان×كروه معنادار بود (جدول

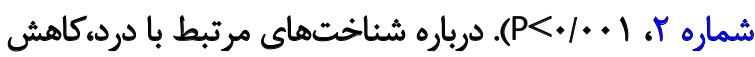

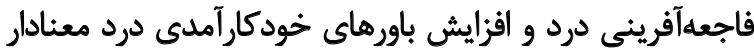

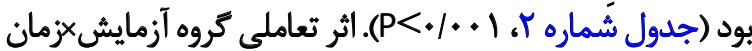

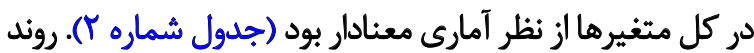

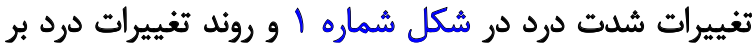

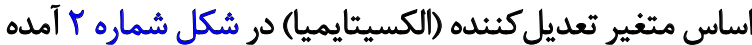
است. - است مت

همان كونه كه در جدول شماره Y مشاهده مي دوده، ميانگين

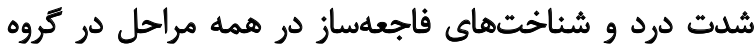

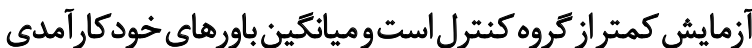

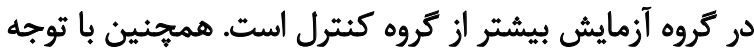

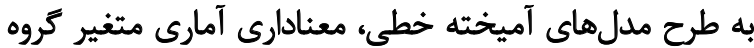

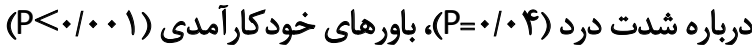

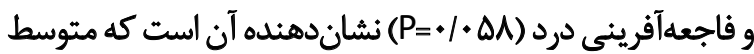

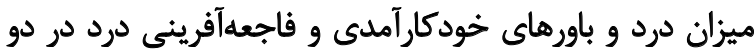
كروه با هم متفاوت است. معنادارى متغير زمان نشاندهنده آن است كه متوسط ميزان

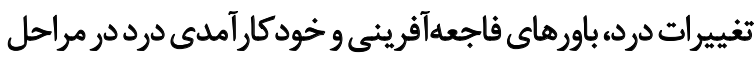

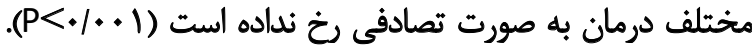

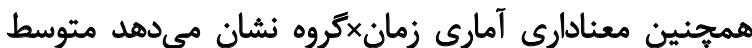

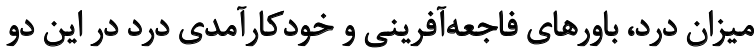

أمره صفر و به باسخ هميشه نمره f تعلق مى تيرد. اعتبار سازه

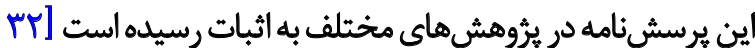

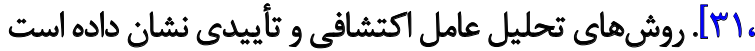

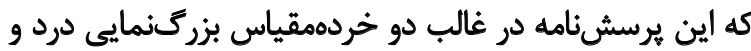

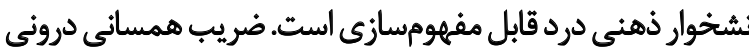

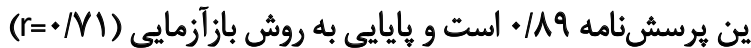

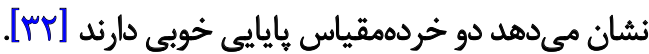

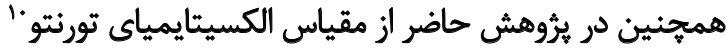

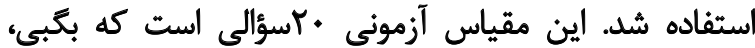

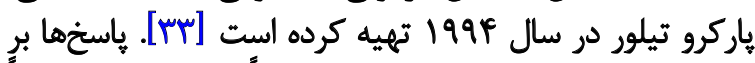

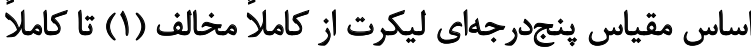

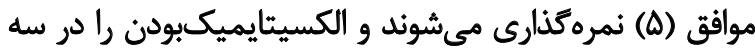

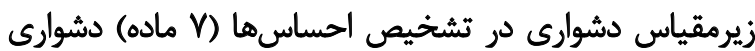

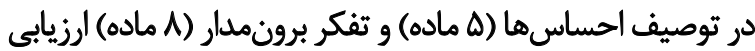

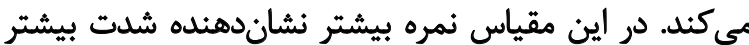

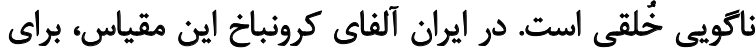

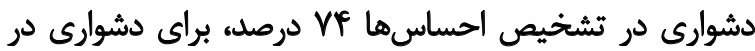

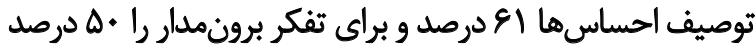

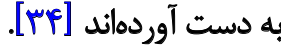
اعتبار كل مقياس در نمونه ايرانى با استفاده از روش دوش

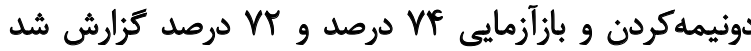

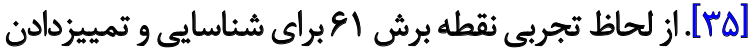

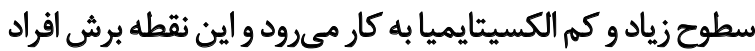

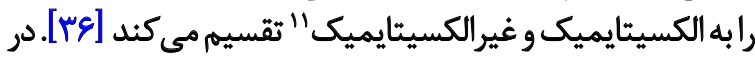

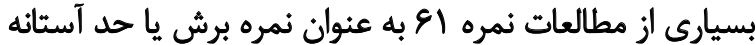

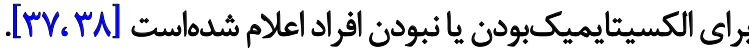

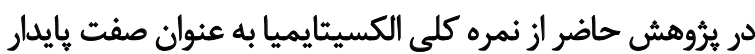
شخصيتى استفاده شده است.

در اين مطالعه نتايج درمان در جهار نقطه زمانى در دو كروه

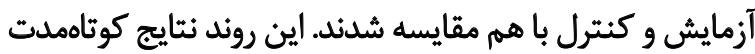

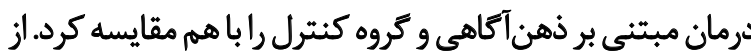

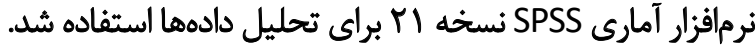

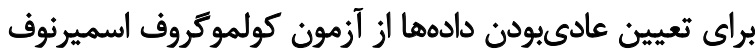

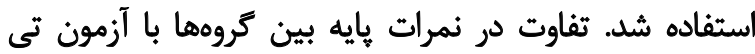
مستقل يا كاى دو اندازهكيرى شد. براي ارزيابى اثربخشى در نمرات شدت درده، خودكارآمدى و

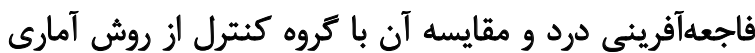

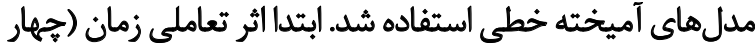

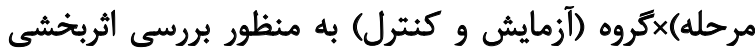

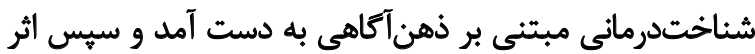

10. Toronto Alexithymia Scale (TAS)

11. Alexithymic or non-alexithymic 
جدول ا. خصوصيات جمعيتشناختياجتماعي و باليني مرتبط با سردرد

\begin{tabular}{|c|c|c|c|c|}
\hline مروه شاهد (ع بنفر) & مروه مداخله (TA نفر) & كل نمونه (rاר نفر) & \multicolumn{2}{|c|}{ مشحخصات بالينى وجمعيتششناختى } \\
\hline TNTIT/NA) & $r q N+(q / q q)$ & $r V / \pi F(1 \cdot / \pi)$ & ميانكين (انحراف استاندارد) & سن (سال) \\
\hline sVIst & 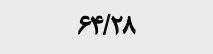 & 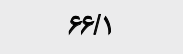 & زن & \multirow{2}{*}{ جنس (درصد) } \\
\hline Tr/TQ & $r \Delta / M$ & $\pi / q$ & هرد & \\
\hline PMIV & Eg/ET & rr/s & 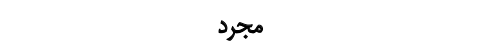 & \multirow{2}{*}{ وضعيت تأهل (درصد) } \\
\hline QNAT & $\Delta r / \Delta V$ & $\Delta \% / \Delta$ & متأهل & \\
\hline SINF & $\varepsilon \cdot M$ & $8 / / \pi$ & 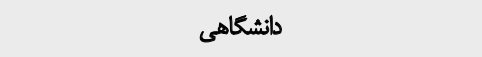 & \multirow{2}{*}{ سطح تحصيلى (درصد) } \\
\hline rNT & TNTA & rNV & غيردانشكاهيى & \\
\hline PI/T & $\Delta H / \Delta r$ & $\varphi / A$ & اشتغال يارووقت يا كلى & \multirow{5}{*}{ وضعيت شغلى (درصد) } \\
\hline NAT & $1 . M$ & QN & بازئشسته & \\
\hline PVIV & $r \Delta / \ldots$ & $\pi / 9$ & 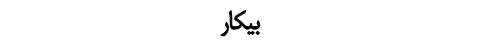 & \\
\hline$r / 9 F$ & V/lf & $r / A$ & خانهدار & \\
\hline$\Delta / M$ & $r / \Delta V$ & $F / \wedge$ & ناتوان از كار به علت درده بيمارى يا ديكر موارد & \\
\hline$r / P(\cdot / 9 Y)$ & $r / \Delta(1 / .9)$ & $r / F V(V / P r)$ & تعلداد (دصرد) & أنكيزه شركت كنئه \\
\hline$r \Delta / r q$ & rq/TA & $\mathrm{rV} / \mathrm{I}$ & ميكرن (با وررا يا بدون اورا) & \multirow{5}{*}{ نوع سردرد الوليه } \\
\hline EM/M & Tr/AS & 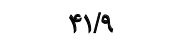 & أنواع سردرد تنشى & \\
\hline$I F / N$ & $1 . M$ & $1 r / 9$ & سردرد كلاستر & \\
\hline raY & $\%$ & ve & همى كرانياى يروكسيمال دورهاى & \\
\hline$\Delta / M$ & V/If & 910 & حملات سردرد نورالزيكى يكىطرفه كوثاهمدت & \\
\hline$I V / T A(F / T V)$ & IV/TAPY/.F) & $I V / \pi T(f / 19)$ & ميانكين (انحراف إستاندارد) & زمان آغاز بيمارى \\
\hline m/TO & $r \Delta / .$. & rq & فيبروميالزيا & \multirow{6}{*}{ ييمارى همايثذ با سردرد مزمن } \\
\hline SINE & er/As & $\Delta r / T$ & كمردرد & \\
\hline PV/T & $r T / \Lambda \Delta$ & 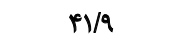 & درد مردن & \\
\hline rT/QT & $I F / T A$ & $19 / \%$ & بيمارى هاى اللثهابى مفاصل (آرثريت روماتوئيد، لويوس و & \\
\hline rV/Y & rM/TH & س & اختلالات توالرشى (الثهاب رودهه IBS و ...) & \\
\hline $\mathbb{1 F T A}$ & $10 / \pi$ & $1 r / 9$ & ديكر موارد & \\
\hline $\operatorname{Ir}(f \in / 4 T)$ & $I V(\varepsilon \cdot M)$ & rq(FNA) & هاييرالكسيتايميك & \multirow{2}{*}{ الكسيتايميا، تعداد (درصد) } \\
\hline$I V(\Delta r / / T)$ & IO(F\&/AV) & $r(\Delta r / T)$ & هييوالكسيثايميك & \\
\hline
\end{tabular}

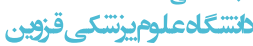

نمرات خام شدت درد در شكل شماره ا حاكى از كاهش تقريباً

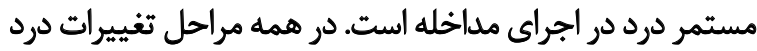

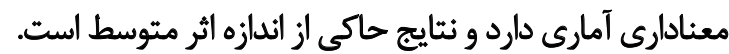

سيس، به منظور ارزيابى روند تغييرات شدت درد در هر مرحله،

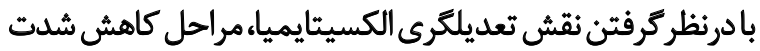

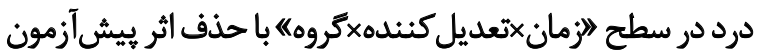

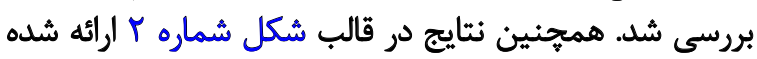

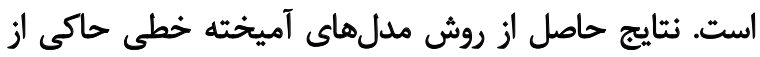

كروه در زمانهاي مختلف متفاوت از يكديكر است (1 + • (P<).

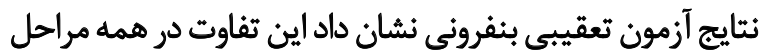

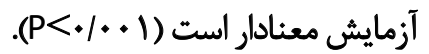

در متغير شدت درد اندازه اثر دى كوهن در حالت كلى

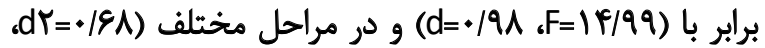

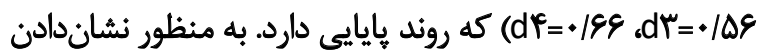

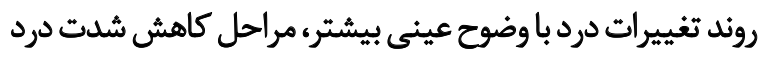

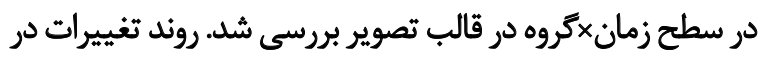


جدول r. ميانكين و انحراف معيار متغيرهاى يُؤوه در هشت هفته به تفكيك كروه آزمايش و كنترل و بررسى اثربخشى مداخله

\begin{tabular}{|c|c|c|c|c|}
\hline يرسشنامه PSEQ & PCS Pيرسثنه & مقياس NRS & & \multirow{2}{*}{ مراحل اندازهئيرى } \\
\hline باورهاي خود كار آمدى درد & فاجعه|آفرينى درد & شدت درد & & \\
\hline$r \Delta / V \Delta \pm 1 / \cdot r$ & PQVADI/qP & 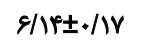 & أرمايش & \multirow[b]{2}{*}{ اول } \\
\hline 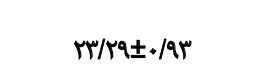 & $\nabla \checkmark / \backslash \pm \backslash / V E$ & $\Delta / \Delta \Delta \pm+/ 1 \varnothing$ & كترل & \\
\hline$r V / / V \pm V / \cdot 1$ & $r N \cdot v \pm 1 / \Lambda_{0}$ & $\Delta /|V \pm \cdot / T|$ & آزمايش & \multirow[b]{2}{*}{ دوم } \\
\hline$M T / Q A \pm \cdot / Q Y$ & rV/retVIFT & $\Delta / \Delta Y \pm * / / q$ & 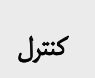 & \\
\hline$T V / Y) \pm \cdot / 9 Y$ & $\operatorname{re} / g \mathrm{v} \pm \mathrm{V} / \mathrm{v}$ & $F / \Lambda F \pm * / T$ & آزمايش & \multirow[b]{2}{*}{ سوم } \\
\hline$r / N^{\top} \pm \cdot / M$ & rg/ATEV/AF & $\Delta / F \vee \pm . / M A$ & كترل & \\
\hline $\begin{array}{l}\text { TNGE } \pm / .1 \\
\text { TH/IVE./QT }\end{array}$ & $\begin{array}{l}r T / 1+ \pm 1 / a r \\
r g / 1 P \pm 1 / \Delta r\end{array}$ & $\begin{array}{l}F / r \Lambda \pm * / 19 \\
\Delta / 8+ \pm * / 1 \gamma\end{array}$ & كتّرل & جهارم \\
\hline $\begin{array}{l}<+1 .+1 \\
\mid \& / 48\end{array}$ & $\begin{array}{l}.1+\Delta A \\
H / E T\end{array}$ & $\begin{array}{l}.1 .4 \\
T / 4\end{array}$ & $\begin{array}{l}\text { P } \\
\text { F }\end{array}$ & - مروه \\
\hline $\begin{array}{l}<+1.01 \\
\text { prepr }\end{array}$ & $\begin{array}{l}<\cdot \mid \cdot+1 \\
8 \mid(A \mid\end{array}$ & $\begin{array}{l}<.1 .01 \\
\text { meN. }\end{array}$ & $\begin{array}{l}\text { P } \\
\text { F }\end{array}$ & زمان \\
\hline $\begin{array}{l}<+1 .+1 \\
r+M r\end{array}$ & $\begin{array}{l}<.1 * .1 \\
f * / 11\end{array}$ & $\begin{array}{l}<.1 .01 \\
17 / 99\end{array}$ & $\begin{array}{l}P \\
F\end{array}$ & تعامل زمان xكروه \\
\hline
\end{tabular}

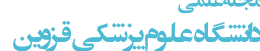

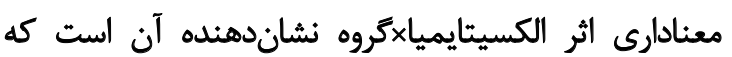

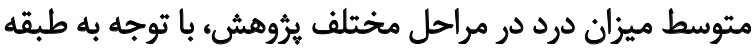

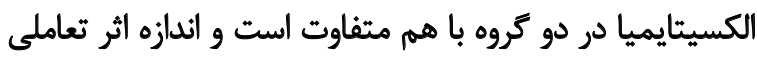

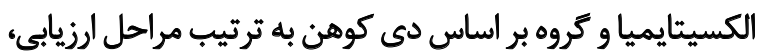

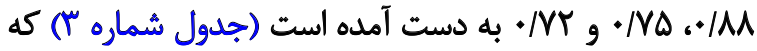

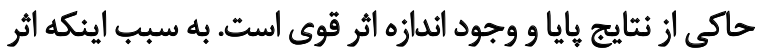

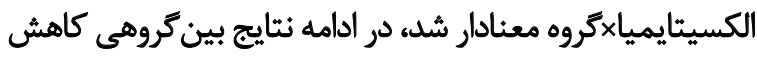

معناداربودن اثر تعديل كنيند مي الكسيتايميا در تعامل با كروه ودر

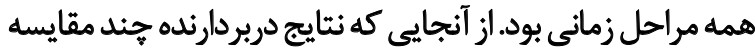

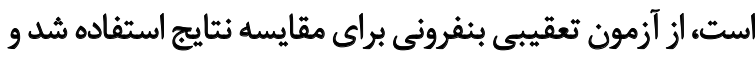

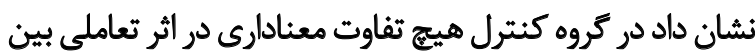

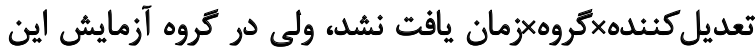

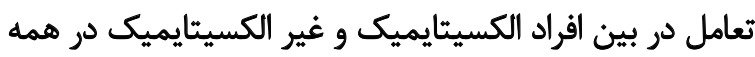

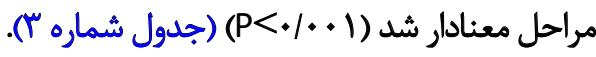

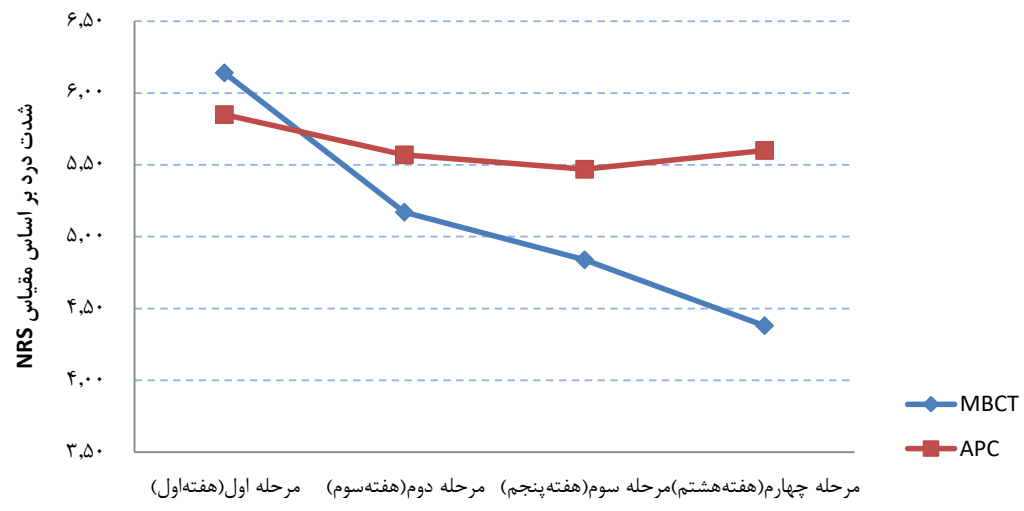

شكل ا. موند تغييرات نمرات خام شدت درد از خط يايه نا انتهاى هشت جلسه (اندازهكيرى در جهار مرحله) 
جدول ب. ميائكين نمرات درد در دو كروه در دفعات متوالى سنجش در بيماران و با حذف اثر بيش آزمون به همراه نتّايج آزمون تعقيبى

\begin{tabular}{|c|c|c|c|c|}
\hline \multicolumn{3}{|c|}{ 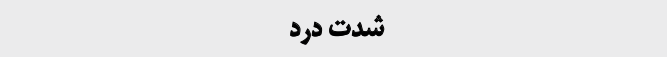 } & \multicolumn{2}{|r|}{ مثغير بررسى شده } \\
\hline مرحله سوم تا جهارم & مرحله دوم تا سوم & مرحله اول تا دوم & & سطوح ارزيابى \\
\hline$r / V F \pm . / 1 F$ & $F / / 9 \pm . / / Y$ & $r / \Delta A \pm . / M$ & الكسيئايميك & \\
\hline$\Delta / 1 \Delta \pm . / 1 \varnothing$ & $\Delta / \Delta \cdot \pm \cdot / V$ & $\Delta / V A \pm . / 1 Y$ & غير الكسيثايميك & 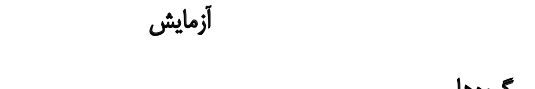 \\
\hline$\Delta / T T \pm . / 1 \%$ & $\Delta / * q \pm+/ / V$ & $\Delta / M \pm \cdot / M$ & المسيتايميك & 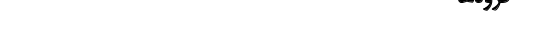 \\
\hline 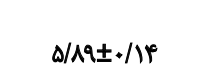 & $\Delta / \Lambda \cdot \pm \cdot / \Delta$ & $\Delta / \& \& \pm . / N$ & غير الكسيتايميك & 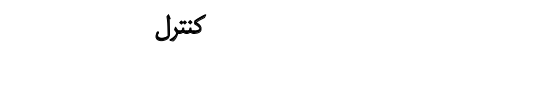 \\
\hline$<\cdot 1 . .1$ & $<\cdot / * .1$ & $<\cdot 1 . .1$ & $P$ & \\
\hline NII & $N N^{*}$ & $\mid r / I F$ & $\mathrm{~F}$ & الكسيتايميا×كروه \\
\hline$\cdot M$ & . NA &.$/ M$ & دى كوهن & \\
\hline$<* / . \bullet 1$ & $<+1+* 1$ & $<+1 \cdot .1$ & اززمايش & \\
\hline$+1 \cdot \mathrm{W}$ &.$/ 199$ & $+/ A 11$ & كنترل & تئيجالزّمون تعقييى يين اقراد اللكسيثايميك و غير الكسيتايميك \\
\hline
\end{tabular}

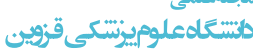

آمارى به دست آوردهاند، ولى نسبت به افراد الكسيتايميك بعد

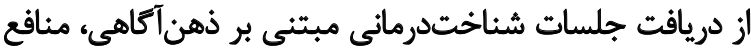
درمائى محدودترى حاصل كردهاند.

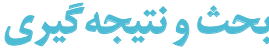

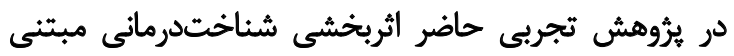

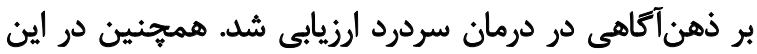

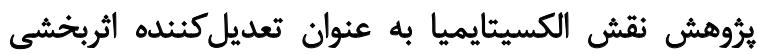

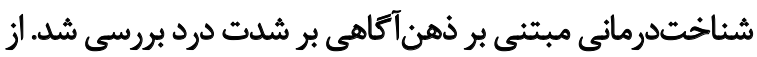

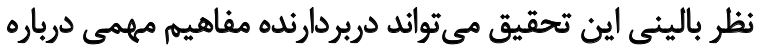

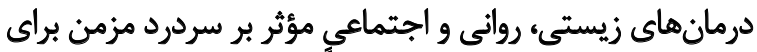

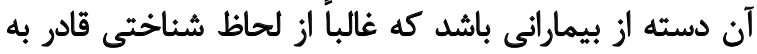

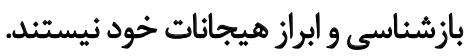
در زمينه جِكونكى تغيير شدت درد در طول زمان، تحقيق
درد با در نظر داشتن اثر تعاملى الكسيتايميا×كروه×زمان شكل شماره Y ترسيهم شده است.

مطابق با جدول شماره ז، فقط در گروه آزمايش تعامل

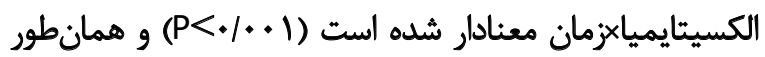

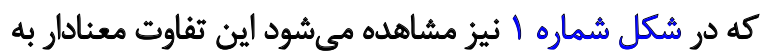

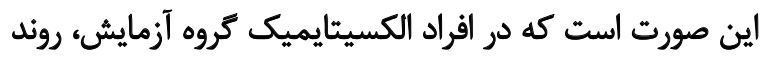

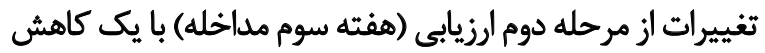

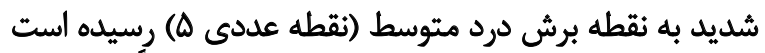

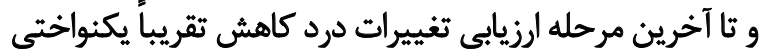

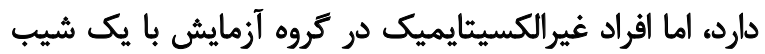

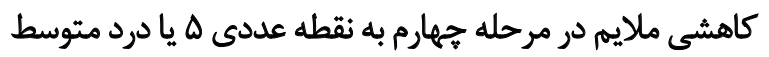

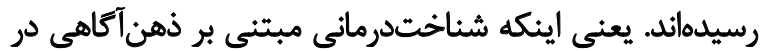

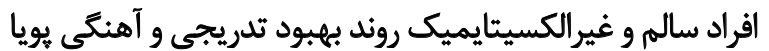
هارد و با اينكه اين افراد نسبت به كروه كنترل نتايج معنادار

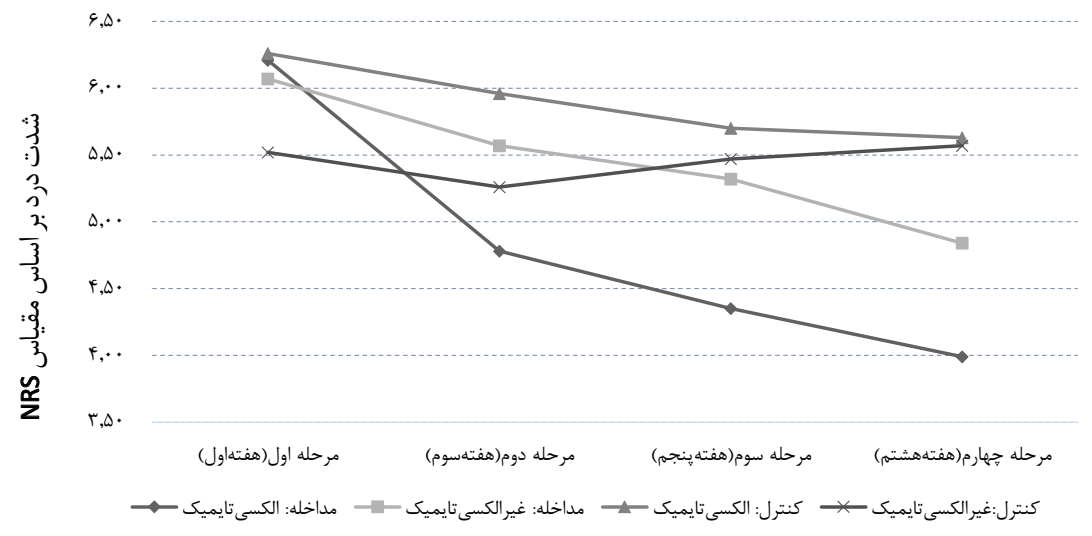




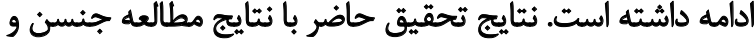

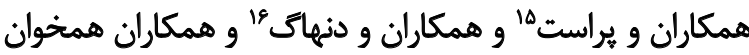

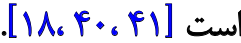

جنين نتايجى در بيماران مبتلا به سردرد مزمن از اين نظر

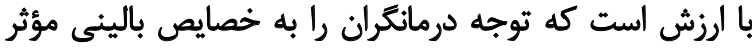

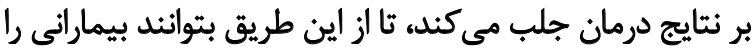

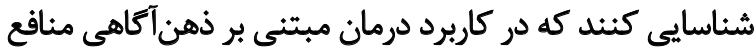

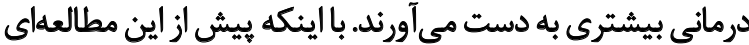

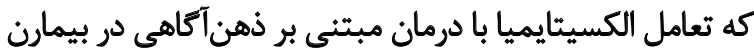

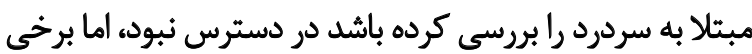

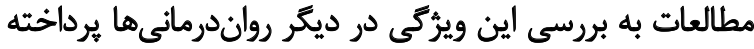

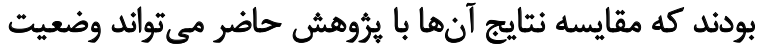

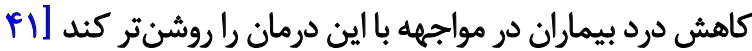

[IA, F.。

يراست" و همكاران در كارآزمايى بالينى خود به بررسى نقش

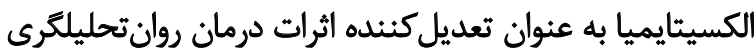

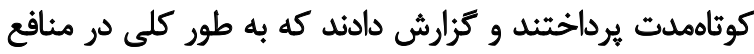

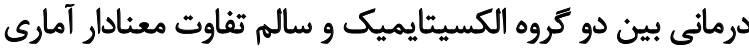

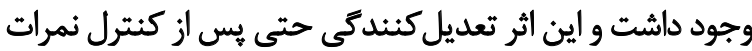

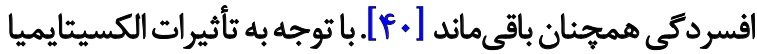

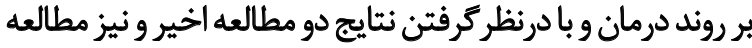

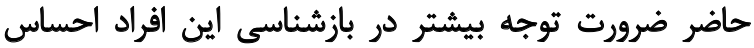

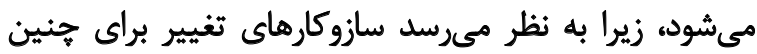
بيمارانى منافع درمانى بيشترى به همراه دارد.

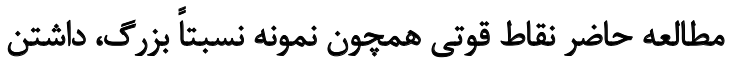

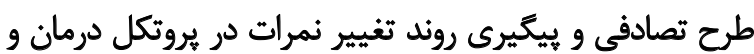

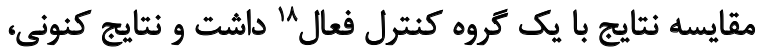

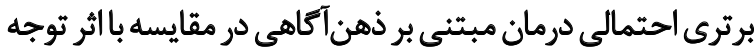

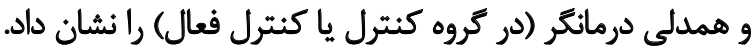

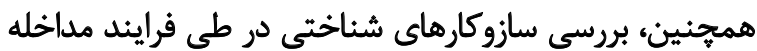

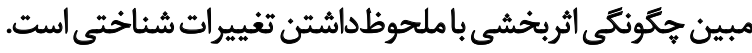

از نقاط ضعف برؤشش حاضر مى توان ريزش نامتعادل بين افراد

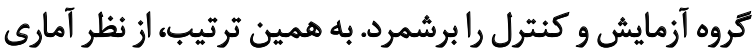

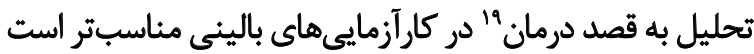

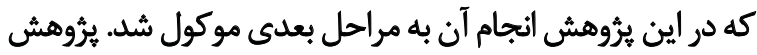

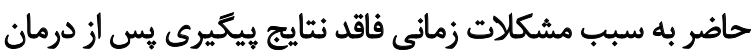

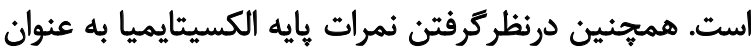

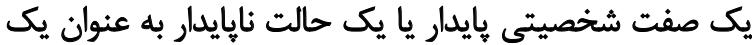

\section{Probst}

16. Dennhag

17. Probst

18. Attention Placebo Control (APC)

19. Intention-To-Treat analysis (ITT)
حاضر نشان داد ميانگين نمرات شدت درد در بيماران كروه

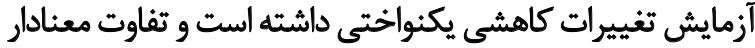

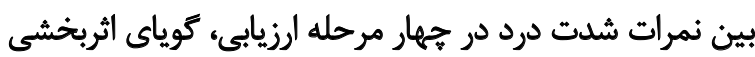

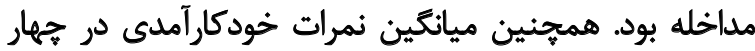

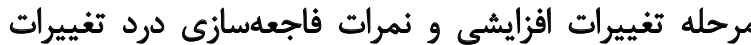

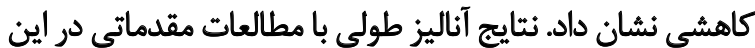

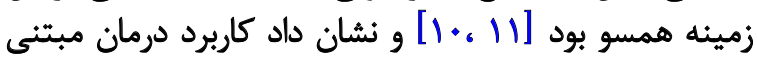

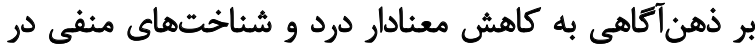

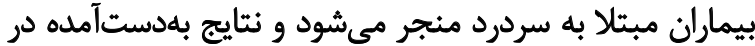

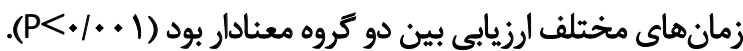

تغييرات معنادار متغيرهاى شناختى باورهاى خودكارآمدى

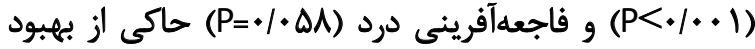

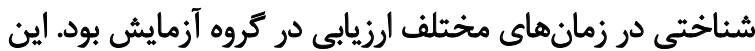

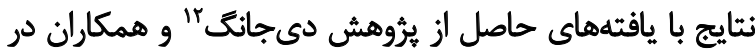

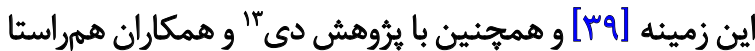

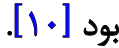

با اين حال هنكام تفسير نتايج و با توجه به اندازههاي اثر درج

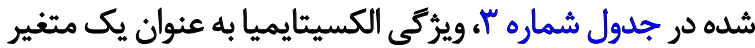

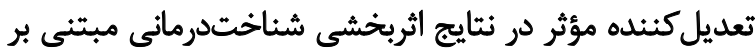

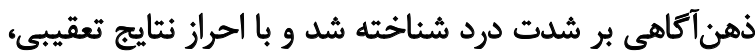

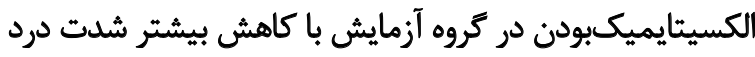

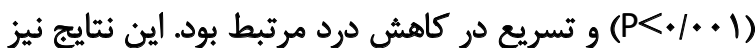

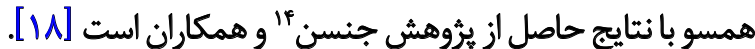

در اين يروهش افراد الكسيتايميك در مقايسه با افراد

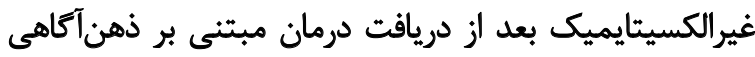

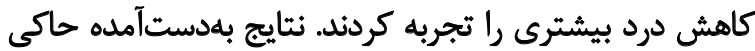

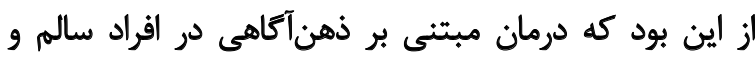

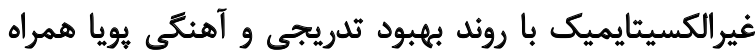

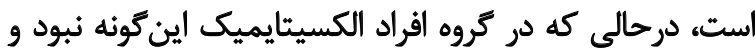

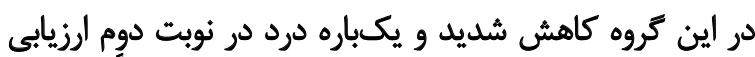

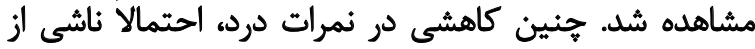

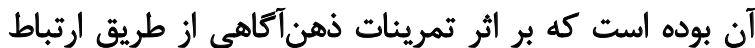

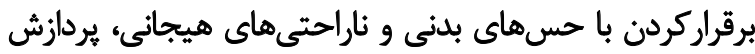

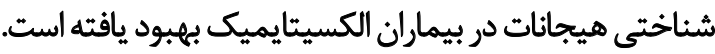

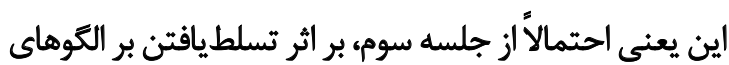

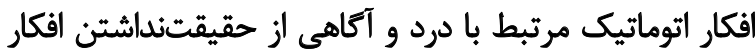

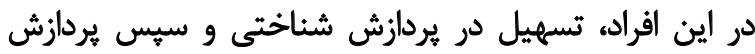

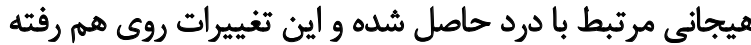

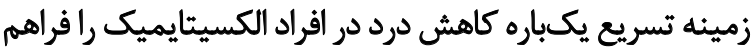

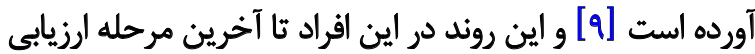

12. de Jong

13. Day

14. Jensen 
جالش مطرح شده است. از ديكر محدوديتهاى مطالعه حاضر براي

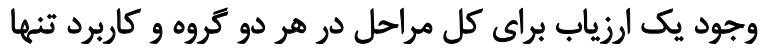

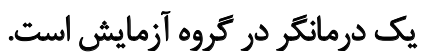

در يك نتيجهيرى كلى، از لحاظ آمارى و بالينى اثرات

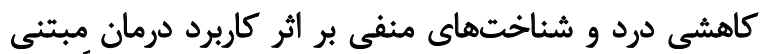

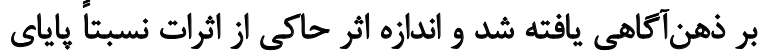

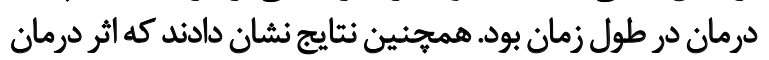

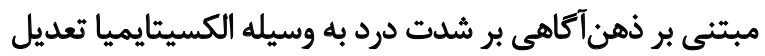

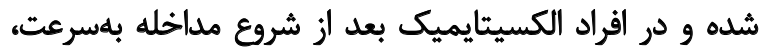

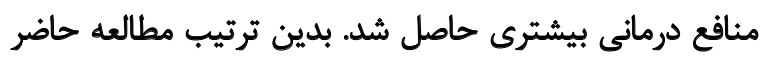

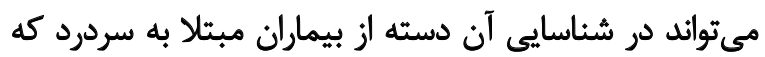

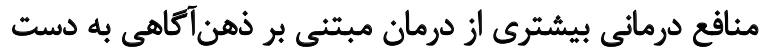
مي آورند ارزشمند باشيد.

مالاحظات أخالاقي

يبيروى ازٔ الصول الخلاق بئوهش

كميته اخلاق يزشكى دانشكاه آزاد اسلامى واحد كرج با كد

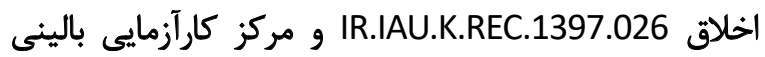
ايران با كد ائ اين مطالعه را ثبت إليت و تأييد كردماند.

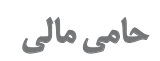

دانشعاه آزاد اسلامى، واحد كرج از اين بروهش حمايت مالى

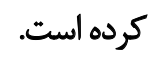

$$
\text { مشاركث نويسندكان }
$$

مفهومسازى: سارا نامجو، محمدرضا صيرفى؛ تحقيق و بررسى:

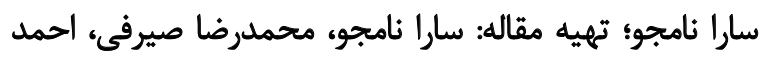

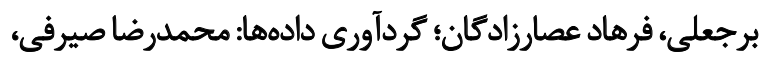

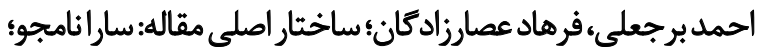

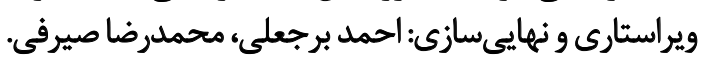

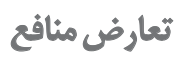

بنابر اظهار نويسندكان، اين مقاله تعارض منافع ندارد.

$$
\text { سوياسكّزازى }
$$

بدينوسيله از همكارى و مساعدت معاونت محترم يروهشى و

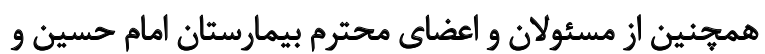
همكاران اجرايى، قدردانى مىشود. 


\section{References}

[1] Cuadrado ML, López-Ruiz P, Guerrero ÁL. Nummular headache: An update and future prospects. Expert Rev Neurother. 2018; 18(1):9-19. [DOI:10.1080/14737175.2018.1401925] [PMID]

[2] Schytz HW, Schoonman GG. Human models of primary headaches. In: Ashina M, Geppetti P, editors. Pathophysiology of Headaches. Berlin: Springer; 2015.

[3] Melzack R. Evolution of the neuromatrix theory of pain. Pain Pract. 2005; 5(2):85-94. [DOI:10.1111/j.1533-
[ 2500.2005.05203.x] [PMID]

[4] Thorn BE. Cognitive therapy for chronic pain: A step-by-step guide. New York: Guilford; 2017.

[5] Hilton L, Hempel S, Ewing BA, Apaydin E, Xenakis L, Newberry $S$, et al. Mindfulness meditation for chronic pain: Systematic review and meta-analysis. Ann Behav Med. 2016; 51(2):199213. [DOI:10.1007/s12160-016-9844-2] [PMID] [PMCID]

[6] Masumian S, Shairi MR, Hashemi M. The effect of mindfulness-based stress reduction on quality of life of the patients with chronic low back pain. Anesth Pain. 2013; 4(1):25-37. [In Persian]

[7] Zare H, Shahriari H, Zare M. Effect of mindfulness-based stress reduction on students' migraine intensity. J Zabol Univ Med Sci. 2014; 6(3):30-9. [In Persian]

[8] Wells RE, Burch R, Paulsen RH, Wayne PM, Houle TT, Loder E. Meditation for migraines: A pilot randomized controlled trial. J Head and Face Pain. 2014; 54(9):1484-95. [DOI:10.1111/ head.12420] [PMID]

[9] Day MA. Mindfulness-Based Cognitive Therapy for chronic pain: A clinical manual and guide. Hoboken: John Wiley \& Sons; 2017. [DOI:10.1002/9781119257875]

[10] Day MA, Thorn BE, Ward LC, Rubin N, Hickman SD, Scogin F, et al. Mindfulness-Based Cognitive Therapy for the treatment of headache pain: A pilot study. Clin J Pain. 2014; 30(2):15261. [PMID]

[11] Day MA, Thorn BE, Rubin NJ. Mindfulness-Based Cognitive Therapy for the treatment of headache pain: A mixed-methods analysis comparing treatment responders and treatment non-responders. Complement Ther Med. 2014; 22(2):278-85. [DOI:10.1016/j.ctim.2013.12.018] [PMID]

[12] Bagby RM, Quilty LC, Segal ZV, McBride CC, Kennedy SH, Costa Jr PT. Personality and differential treatment response in major depression: A randomized controlled trial comparing cognitive-behavioural therapy and pharmacotherapy. Canad J Psychiatry. 2008; 53(6):361-70. [DOI:10.1177/070674370805 300605] [PMID] [PMCID]

[13] Turner JA, Holtzman S, Mancl L. Mediators, moderators, and predictors of therapeutic change in cognitive-behavioral therapy for chronic pain. Pain. 2007; 127(3):276-86. [DOI:10.1016/j. pain.2006.09.005] [PMID]

[14] Hosoi M, Molton IR, Jensen MP, Ehde DM, Amtmann S, $\mathrm{O}$ 'Brien $\mathrm{S}$, et al. Relationships among Alexithymia and pain intensity, pain interference, and vitality in persons with neuro- muscular disease: Considering the effect of negative affectivity. Pain. 2010; 149(2):273-7. [DOI:10.1016/j.pain.2010.02.012] [PMID] [PMCID]

[15] Galli F, Caputi M, Sances G, Vegni E, Bottiroli S, Nappi G, et al. Alexithymia in chronic and episodic migraine: A comparative study. J Mental Health. 2017; 26(3):192-6. [DOI:10.3109/0963 8237.2015.1124404] [PMID]

[16] Lumley MA, Neely LC, Burger AJ. The assessment of Alexithymia in medical settings: Implications for understanding and treating health problems. J Pers Assess. 2007; 89(3):230-46. [DOI:10.1080/00223890701629698] [PMID] [PMCID]

[17] Kabat-Zinn J. Mindfulness-based interventions in context Past, present, and future. Clinical Psychology: Sci Pract. 2003; 10(2):144-56. [DOI:10.1093/clipsy.bpg016]

[18] Jensen-Johansen MB, Christensen $S$, Valdimarsdottir H, Zakowski $S$, Jensen $A B$, Bovbjerg $D$, et al. Effects of an expressive writing intervention on cancer-related distress in Danish breast cancer survivors-Results from a nationwide randomized clinical trial. Psycho-Oncol. 2013; 22(7):1492-500. [DOI:10.1002/ pon.3193] [PMID]

[19] Day MA, Thorn BE. The mediating role of pain acceptance during Mindfulness-Based Cognitive Therapy for headache. Complement Ther Med. 2016; 25:51-4. [DOI:10.1016/j. ctim.2016.01.002] [PMID]

[20] Kraemer HC, Wilson GT, Fairburn CG, Agras WS. Mediators and moderators of treatment effects in randomized clinical trials. Arch Gen Psychiatry. 2002; 59(10):877-83. [DOI:10.1001/ archpsyc.59.10.877] [PMID]

[21] Headache Classification Committee of the International Headache Society. The international classification of headache disorders, (beta version). Cephalalgia. 2013; 33(9):629-808. [DOI:10.1177/0333102413485658] [PMID]

[22] Rains JC, Poceta JS. Headache and sleep disorders: Review and clinical implications for headache management. Headache. 2006; 46(9):1344-63. [DOI:10.1111/j.15264610.2006.00578.x] [PMID]

[23] Haefeli M, Elfering A. Pain assessment. Eur Spine J. 2006 15(1):S17-S24. [DOI:10.1007/s00586-005-1044-x] [PMID] [PMCID]

[24] Dworkin RH, Turk DC, Farrar JT, Haythornthwaite JA, Jensen MP, Katz NP, et al. Core outcome measures for chronic pain clinical trials: IMMPACT recommendations. Pain. 2005; 113(1):9-19. [DOI:10.1016/j.pain.2004.09.012] [PMID]

[25] Hawker GA, Mian S, Kendzerska T, French M. Measures of adult pain: Visual Analog Scale for Pain (VAS Pain), Numeric Rating Scale for Pain (NRS Pain), Mcgill Pain Questionnaire (MPQ), Short-Form Mcgill Pain Questionnaire (SF-MPQ), Chronic Pain Grade Scale (CPGS), Short Form-36 Bodily Pain Scale (SF-36 BPS), and Measure of Intermittent and Constant Osteoarthritis Pain (ICOAP). Arthritis Care Res. 2011; 63(S11):S240-S52. [DOI:10.1002/acr.20543] [PMID]

[26] Serlin RC, Mendoza TR, Nakamura Y, Edwards KR, Cleeland CS. When is cancer pain mild, moderate or severe? Grading pain severity by its interference with function. Pain. 1995; 61(2):277-84. [DOI:10.1016/0304-3959(94)00178-H] 
[27] Nicholas M. Self-efficacy and chronic pain. Ann Conference $\mathrm{Br}$ Psychol Soc. 1989; 2(2):1-7.

[28] Nicholas MK. The pain self-efficacy questionnaire: taking pain into account. Euro J Pain. 2007; 11(2):153-63. [DOI:10.1016/j. ejpain.2005.12.008] [PMID]

[29] Asghari A, Nicholas MK. An investigation of pain self-efficacy beliefs in Iranian chronic pain patients: A preliminary validation of a translated English-language scale. Pain Med. 2009; 10(4):619-32. [DOI:10.1111/j.1526-4637.2009.00623.x] [PMID]

[30] Sullivan MJ, Bishop SR, Pivik J. The pain catastrophizing scale: Development and validation. Psychol Assess. 1995; 7(4):52432. [DOI:10.1037/1040-3590.7.4.524]

[31] Miró J, Nieto R, Huguet A. The Catalan version of the Pain catastrophizing scale: A useful instrument to assess catastrophic thinking in whiplash patients. J Pain. 2008; 9(5):397-406. [DOI:10.1016/j.jpain.2007.12.004] [PMID]

[32] Rahmati N, Moghadam A, Ali M, Shairi M, Paknejad M, Rahmati Z, et al. A study of the psychometric properties of the pain catastrophizing scale amongst Iranian patients with chronic persistent pain. Sci J llam Univ Med Sci. 2017; 25(1):63-79. [DOI:10.29252/sjimu.25.1.63]

[33] Parker JD, Taylor GJ, Bagby RM. The relationship between emotional intelligence and Alexithymia. Pers Individ Dif. 2001; 30(1):107-15. [DOI:10.1016/S0191-8869(00)00014-3]

[34] Ghorbani N, Bing MN, Watson P, Davison HK, Mack DA. Self-reported emotional intelligence: Construct similarity and functional dissimilarity of higher-order processing in Iran and the United States. Int J Psych. 2002; 37(5):297-308. [DOI:10.1080/00207590244000098]

[35] Besharat MA. Reliability and factorial validity of a Farsi version of the 20-item Toronto Alexithymia scale with a sample of Iranian students. Psychol Rep. 2007; 101(1):209-20. [DOI:10.2466/ PR0.101.5.209-220] [PMID]

[36] Taylor GJ, Bagby RM, Ryan DP, Parker JD, Doody KF, Keefe P. Criterion validity of the Toronto Alexithymia scale. Psychosom Med. 1988; 50(5):500-9. [DOI:10.1097/00006842-19880900000006] [PMID]

[37] Honkalampi K, Hintikka J, Laukkanen E, Viinamäki JLH. Alexithymia and depression: A prospective study of patients with major depressive disorder. Psychosom. 2001; 42(3):229-34. [DOI:10.1176/appi.psy.42.3.229] [PMID]

[38] Franz M, Popp K, Schaefer R, Sitte W, Schneider C, Hardt J, et al. Alexithymia in the German general population. Social Psychiatry and Psychiatric Epidemiol. 2008; 43(1):54-62. [DOI:10.1007/s00127-007-0265-1] [PMID]

[39] de Jong M, Lazar SW, Hug K, Mehling WE, Hölzel BK, Sack AT, et al. Effects of Mindfulness-Based Cognitive Therapy on body awareness in patients with chronic pain and comorbid depression. Front Psychol. 2016; 7:967. [DOI:10.3389/fpsyg.2016.00967] [PMID] [PMCID]

[40] Probst T, Sattel H, Gündel H, Henningsen P, Kruse J, Schnei$\operatorname{der} \mathrm{G}$, et al. Moderating effects of Alexithymia on associations between the therapeutic alliance and the outcome of brief psychodynamic-interpersonal psychotherapy for multisomatoform disorder. Front Psychiatry. 2017; 8:261. [DOI:10.3389/ fpsyt.2017.00261] [PMID] [PMCID]

[41] Dennhag I, Ybrandt H, Sundström A. The relationship between clients' personality traits, working alliance and therapy. Curr Issues in Personal Psychol. 2017; 5(2):132-42. [DOI: 10.5114/cipp.2017.65244] 